\title{
Accurate Positioning for Intervention on the Beating Heart using a Crawling Robot
}

\author{
N.A. Patronik ${ }^{1}$, T. Ota $^{2}$, M.A. Zenati ${ }^{1,2}$, and C.N. Riviere ${ }^{1}$ \\ ${ }^{1}$ The Robotics Institute, Carnegie Mellon University, Pittsburgh, PA, USA \\ ${ }^{2}$ Division of Cardiothoracic Surgery, University of Pittsburgh, Pittsburgh, PA, USA
}

\begin{abstract}
Heart failure resulting from myocardial infarct, oxygen-deprived tissue death, is a serious disease that affects over 20 million patients in the world. The precise injection of tissue-engineered materials into the infarct site is emerging as a treatment strategy to improve cardiac function for patients with heart failure. We have developed a novel miniature robotic device (HeartLander) that can act as a manipulator for precise and stable interaction with the epicardial surface of the beating heart by mounting directly to the organ. The robot can be delivered to and operate within the intrapericardial space with the chest closed, through a single small incision below the sternum. The tethered crawling device uses vacuum pressure to maintain prehension of the epicardium, and a drive wire transmission motors for actuation. An onboard electromagnetic tracking sensor enables the display of the robot location on the heart surface to the surgeon, and closed-loop control of the robot positioning to targets. In a closed-chest animal study with the pericardium intact, HeartLander demonstrated the ability to acquire a pattern of targets located on the posterior surface of the beating heart within an average of $1.7 \pm 1.0 \mathrm{~mm}$. Dye injections were performed following the target acquisitions to simulate injection therapy for heart failure. HeartLander may prove useful in the delivery of intrapericardial treatments, like myocardial injection therapy, in a precise and stable manner, which could be performed on an outpatient basis.
\end{abstract}

\section{INTRODUCTION}

$\mathrm{H}$ eart failure, the inability of the heart's pumping function to keep up with the demands of the body, affects more than 20 million patients in the world, with approximately 400,000 newly diagnosed patients and 50,000 deaths annually [1]. This disease is typically caused by myocardial infarction, the death of heart muscle tissue from oxygen starvation, resulting from the narrowing or blockage of one of the coronary arteries that supply the myocardium with blood. Although there is currently no known cure for heart failure, the injection of tissue-engineered materials (e.g., stem cells or biopolymers) into the infarct area to improve heart function is emerging as a therapeutic strategy for post-myocardial infarct heart failure [2-5]. Myocardial injection therapy is currently dominated by catheter-based approaches that access the inside of the heart through small incisions into the larger vasculature of the arm or leg.

Manuscript received April 23, 2008. This work was supported by the National Science Foundation (grant no. EEC-9731748) and a NASA GSRP Fellowship (NNG05GL63H).
Although this transcatheter technique has low associated access morbidity, there are several advantages to performing the injections directly into the outside epicardial surface of the heart: clear detection of target infarct lesions, decreased likelihood of cerebrovascular complications [6], and superior site specific efficacy [7]. The major drawback to direct epicardial injection therapy is the lack of dedicated minimally invasive access technology, causing it to be performed only in conjunction with other procedures requiring a full sternotomy or thoracotomy. These invasive approaches incur high associated morbidity, despite the intrinsically simple and noninvasive nature of the injection procedure. The DaVinci ${ }^{\mathrm{TM}}$ robotic surgical system (Intuitive Surgical, Mountain View, CA) could be used for minimally invasive epicardial injection [8], but it requires multi-port placement and lung deflation, and does not readily facilitate the precise control of injection placement or depth with its rigid endoscopic instrumentation. A dedicated technology for precise and stable interaction with the heart that balances treatment efficacy and minimal invasiveness would benefit direct epicardial injection therapy.

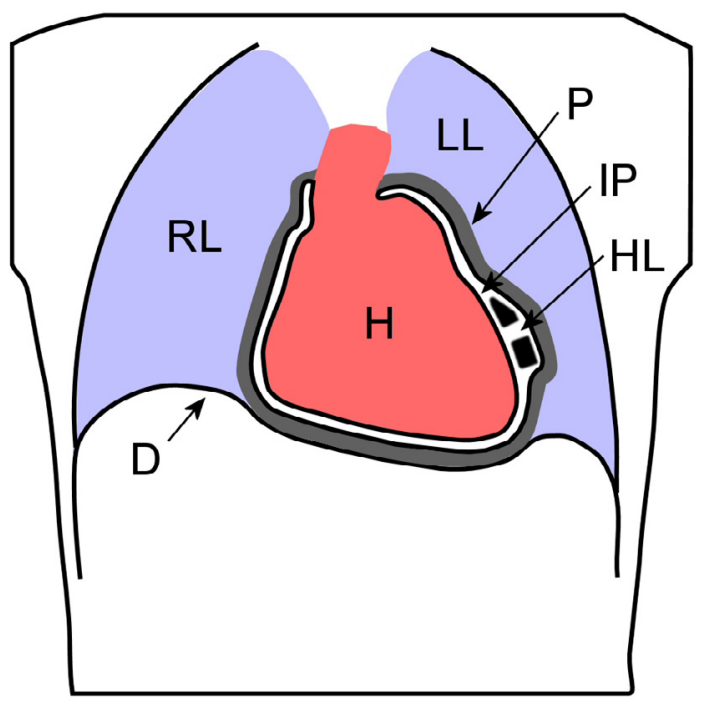

Fig. 1. Coronal illustration of HeartLander $(H L)$ in the intrapericardial space $(I P)$. The surrounding organs include the heart $(H)$, pericardium $(P)$, right lung $(R L)$, left lung $(L L)$, and diaphragm $(D)$. The sizes of the intrapericardial space and pericardium have been greatly exaggerated for clarity. The robot tether is not shown. 


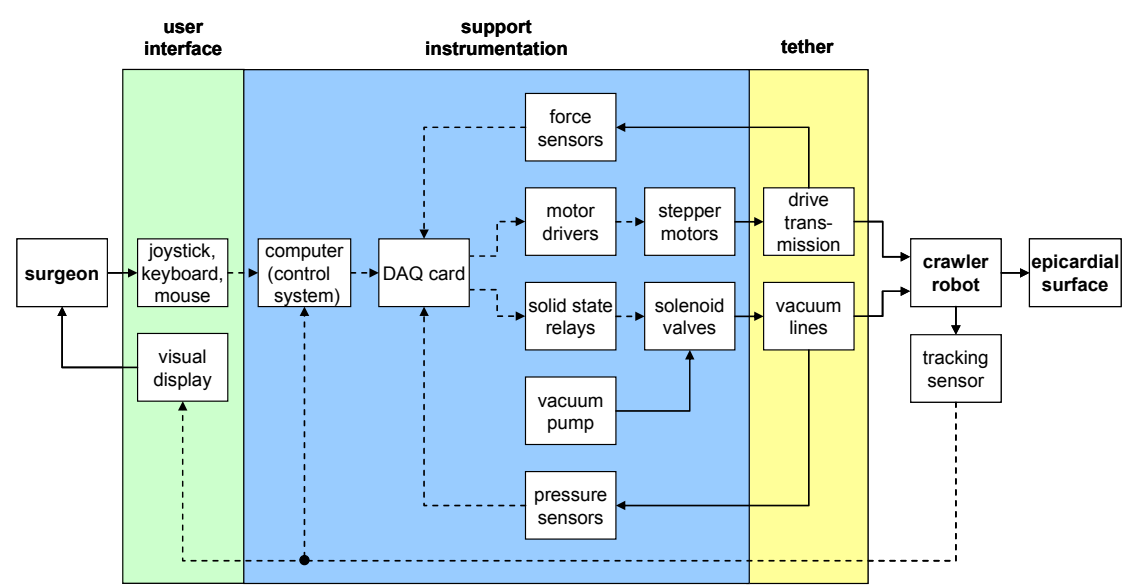

(a)

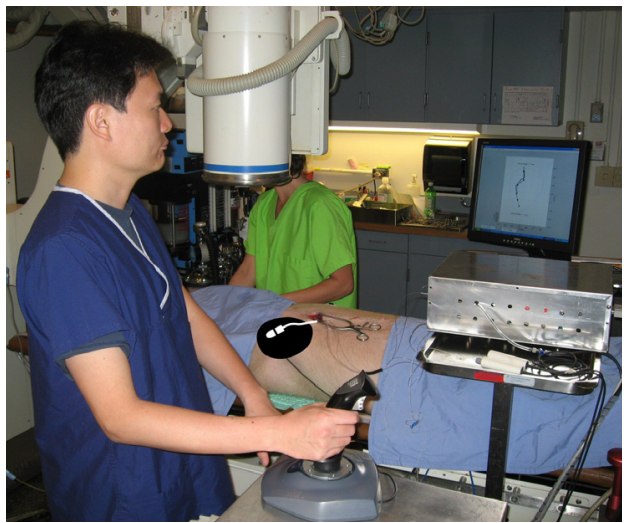

(b)

Fig. 2. (a) Schematic diagram of the HeartLander system, which comprises the user interface, the support instrumentation, and the tethered crawling robot. Mechanical flow is indicated by solid lines, while data flow is shown by broken lines. (b) Photograph of the operating room during animal testing, showing the surgeon interacting with the HeartLander system. The approximate heart shape and robot location inside the body have been illustrated for clarity.

To address this need, we have developed a novel miniature robotic device (HeartLander) that can act both as a mobile robot for navigation and as a manipulator for positioning tasks on the epicardial surface of the beating heart. This device could facilitate the delivery of minimally invasive therapy through a single incision below the sternum. HeartLander operates within the intrapericardial space - the thin fluid layer above the epicardial heart surface and beneath the pericardial sac that encloses the heart (Fig. 1). Previous prototypes have demonstrated safe remotecontrolled navigation over the beating heart $[9,10]$. This paper describes the addition of real-time 3-D localization, a semi-autonomous control system for the acquisition of targets, and an onboard tool for direct epicardial injection into the myocardium. We present an evaluation of these components through the delivery of an injection pattern onto the posterior left ventricle of a beating porcine heart through a single port approach with the chest closed.

\section{RoвOt DESIGN}

\section{A. HeartLander System Overview}

The HeartLander system consists of a surgical user interface, supporting robotic instrumentation, and a tethered crawling device or crawler (Fig. 2). The surgeon interacts with the system through a joystick and standard computer interface, which features a graphic display of the current location of the robot on the heart surface. The support system uses external motors, vacuum pumps, and a computer control system to translate the commands of the surgeon into the appropriate actions of the crawler. The miniature tethered crawling device is the therapeutic end effector of the robotic system, and is located within the intrapericardial space of the patient. This tethered design allows the crawler to be miniature, lightweight, electrically passive, and disposable.
The envisioned intrapericardial therapies that would be facilitated by HeartLander, such as myocardial injection therapy, require multiple treatments to be made at precise locations on the epicardial surface of the heart. In clinical use, the surgeon would first place the crawler on the epicardium through a percutaneous incision below the sternum and subsequent pericardial incision. The robot would then semi-autonomously navigate to the specified target pattern area, and acquire each individual target using a series of positioning motions. A single treatment would be performed by the surgeon after each target acquisition.

\section{B. Crawler Design}

The tethered crawler consists of two bodies (front and rear) that each contain an independent suction pad for prehension of the epicardium using vacuum pressure (Fig. $3 b)$. Each suction pad has a cylindrical shape, with a diameter of $6.0 \mathrm{~mm}$ and a depth of $3.5 \mathrm{~mm}$, which is integrated into the body. Thin latex skirts surround the periphery of the suction pads to help create a vacuum seal with the epicardium. The crawler bodies are each $5.5 \times 8.0 \times$ $8.0 \mathrm{~mm}(\mathrm{H} \mathrm{x} \mathrm{W} \mathrm{x} \mathrm{L})$, and are made of a durable plastic using stereolithography fabrication. These dimensions include a 2$\mathrm{mm}$ diameter working channel, and allow the robot to fit through an $8-\mathrm{mm}$ diameter port. The drive transmission that actuates the crawler is composed of two super-elastic nitinol wires $(0.3 \mathrm{~mm}$ in diameter) that are attached to the front body, and sheathed within lengths of low-friction plastic tubing that are attached to the rear body (Fig. 3). The wires slide freely within the plastic sheaths when driven by the support system motors. The lengths of exposed wire between the body sections $\left(\mathrm{L}_{1}\right.$ and $\mathrm{L}_{2}$ ) determine the distance (L) and angle ( $\beta$ ) between the crawler bodies (Fig. 4). Vacuum pressure is regulated up to $450-600 \mathrm{mmHg}$ by computer-controlled valves in the support system, and is supplied to the suction pads via vacuum lines. 


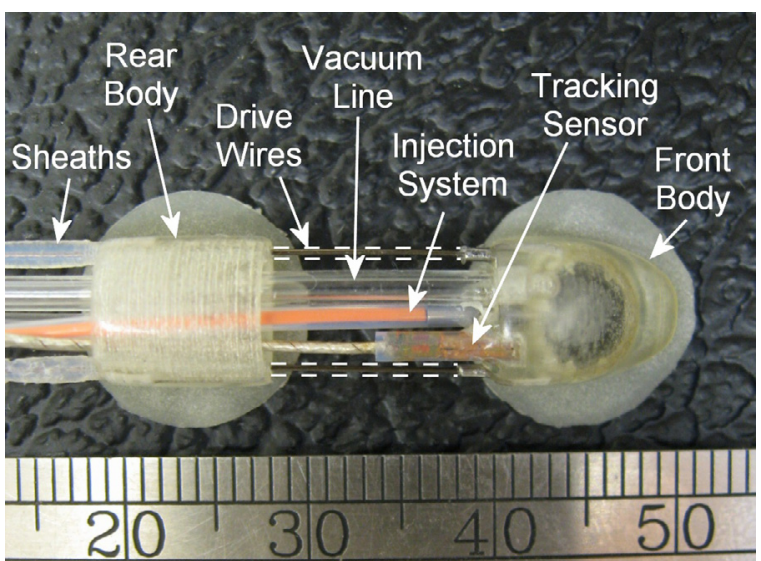

(a)

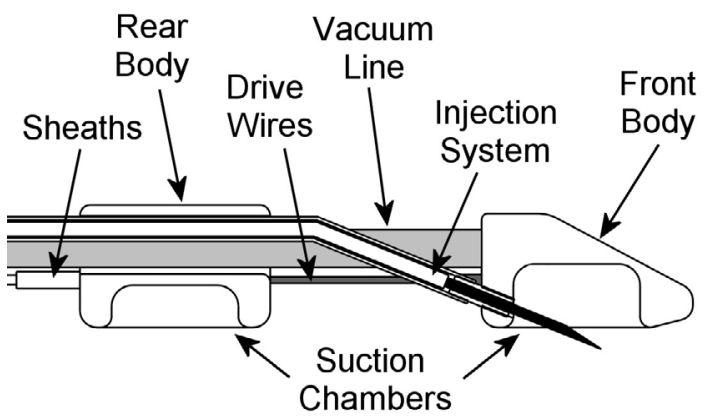

(b)

Fig. 3. (a) Photograph of the top of the HeartLander crawling robot. (b) Illustration of the profile of the crawling robot, with a cutaway along the median coronal plane to show the suction pads and injection system.

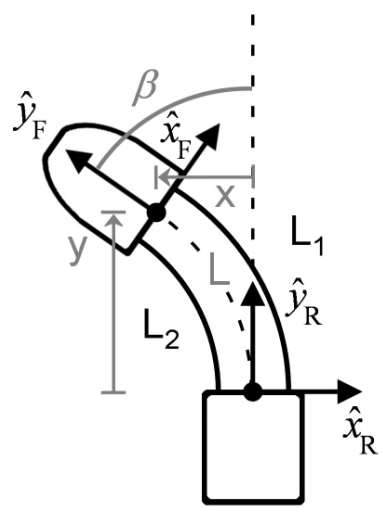

Fig. 4. Illustration of the front crawler body turning to the left, with kinematic axes and variables shown. The Cartesian location $(x, y)$ of the front body with respect to the rear body is controlled by the exposed wire lengths $L_{1}$ and $L_{2}$. The resulting bearing angle $\beta$ is also shown.

The inchworm-like locomotion of the crawler is generated by the computer control system by regulating the wire lengths between the crawler bodies and the vacuum pressure in the corresponding suction pads (for more details, see [9]). The vacuum line pressures and drive wire forces are recorded and used offline in the analysis of locomotion performance. During both navigation and positioning, the inverse kinematics of the crawler are used by the control system to calculate the wire lengths $\left(\mathrm{L}_{1}\right.$ and $\left.\mathrm{L}_{2}\right)$ required to move the front body to the desired Cartesian location $(\mathrm{x}, \mathrm{y})$ with respect to the rear body (Fig. 4). A full development of the inverse kinematics can be found in [11].

\section{Remote Injection System}

A remote injection system for performing myocardial injections from the intrapericardial space has been developed to fit within the working channel of the HeartLander crawler. When the crawler is in motion, the 27 gauge needle is safely housed inside the working channel of the front body. When the crawler front body reaches the desired target location, the needle is extended into the tissue that has been drawn into the active front body suction pad (Fig. 3b). The proximal end of the needle injection system is connected to a syringe. The depth of the needle penetration into the tissue is set by an adjustable mechanical constraint within the range of $1-5 \mathrm{~mm}$.

\section{Electromagnetic Tracking and Localization}

The graphic display and robot localization for control use the data from an electromagnetic tracking system (microBIRD, Ascension Technologies, Burlington, VT) [12]. The position and orientation of a miniature tracking sensor, located on the front body of the crawler (Fig. 3a), are measured in real time with respect to a magnetic transmitter attached to the operating table. This method of tracking does not require a line of sight between the sensor and the transmitter, and is thus well suited for tracking tools located inside the body. The tracking system has a reported static translational and angular accuracy of $1.4 \mathrm{~mm}$ and $0.5^{\circ}$, respectively, within the transmitter workspace. The reported translational and angular resolution are $0.5 \mathrm{~mm}$ and $0.1^{\circ}$, respectively.

The graphic display generates a 2-D projection of the 3-D location of the robot on a geometric representation of the heart surface, from the current view angle specified by the surgeon. The creation of the geometric heart model is described in Section III. B. During the target acquisition task, the 3-D location of the target pattern is also displayed. The surgeon uses the display to select the current target, and monitors the progress of the crawler front body during the acquisition. This display is the only form of visualization during the porcine testing, because the robot is inserted through a small incision with the chest closed.

We wish to localize the robot with respect to the moving reference frame of the beating heart, rather than a traditional stationary reference frame. The crawler naturally lies within this heart-based reference frame because it passively moves with the portion of the epicardium to which it is attached at any given time. The tracking system, however, measures the sensor position and orientation with respect to the stationary magnetic transmitter, thus requiring a transformation to the heart-based reference frame. In order to perform this transformation, we filter the physiological motion out of the 
tracking data in real time. A third-order Chebyshev Type II low-pass filter with a $20-\mathrm{dB}$ cutoff frequency at $1.0 \mathrm{~Hz}$ is used to remove the heartbeat motion from the tracking data. To remove the respiration component of the physiological motion, we then use a series of two second-order IIR notch digital filters, with notch frequencies at the primary $(0.23$ $\mathrm{Hz})$ and secondary harmonics $(0.46 \mathrm{~Hz})$ of the ventilation rate. Notch filters are appropriate for this task, because the respiration rate is precisely set by the ventilator to $0.23 \mathrm{~Hz}$. In offline testing, this filtering method attenuates the tangential physiological motion component by $81 \%$, from $7.3 \pm 1.2 \mathrm{~mm}$ to $1.4 \pm 0.5 \mathrm{~mm}$. The time delay caused by the filtering was $1 \mathrm{~s}$. More details can be found in [11].

\section{E. Positioning Control}

The positioning control system enables the surgeon to acquire each target in the specified pattern, in a semiautonomous manner. The surgeon first defines the number of targets for the pattern, along with their positions relative to on another. The surgeon then specifies the position and orientation of the target pattern on the heart surface model, using the graphic display of the user interface as a reference. The targets are thus fixed in space, and defined with respect to the electromagnetic tracking system. The crawler is then placed on the epicardial surface by the surgeon, and the target acquisition task proceeds. The surgeon selects the current target from the pattern with the user interface, and presses the joystick to begin the acquisition. The control system then uses the crawler inverse kinematics to align the front body and the current target based on the 3-D location of the robot measured from the tracking sensor and the fixed location of the current target. Throughout the target pattern acquisition, the rear body remains fixed to the epicardium unless the control system determines that taking a step toward the current target is necessary. Target acquisition proceeds as long as the surgeon presses the joystick, until the target is acquired within the distance specified by the surgeon though the user interface. The surgeon monitors the progress of the robot toward the each of the targets with the graphic display. This semi-autonomous control paradigm allows the surgeon to control the entire system at a high level, while allowing the computer control system to perform the low-level calculations for the motions for the robot.

\section{EXPERIMENTAL PROTOCOL}

An animal study was performed to evaluate the ability of HeartLander to accurately acquire a target pattern on the epicardium of a porcine beating heart in a semi-autonomous manner. Direct epicardial injection therapy was simulated by injecting dye into the myocardium using the remote injection system, which also required HeartLander to maintain a stable platform for safe interaction with the beating heart.

\section{A. Animal Preparation}

A healthy Yorkshire swine (body weight of $40 \mathrm{~kg}$ ) was anesthetized and placed in a supine position. A small subxiphoid incision $(40 \mathrm{~mm})$ and pericardial incision $(15$ $\mathrm{mm}$ ) were created to access the apex of the left ventricle. Blood pressure and electrocardiogram were continuously monitored through the trials. All testing was performed with the chest closed and pericardium intact through a subxiphoid approach. The vacuum pressure range was maintained within the range of 500 to $550 \mathrm{mmHg}$, which is considered to be safe by the FDA for suction-based mechanical stabilization of the epicardial surface [13].

\section{B. Heart Surface Model Construction}

Prior to testing, a stationary wire frame model of the heart surface was generated for the graphic display of the user interface. This animal-specific geometric model provided a reference to localize the robot on the heart surface during the closed-chest animal testing. Although the surface model did not contain any fine anatomical detail, the surgeon was able to use the shape and size of the model to estimate the general anatomical regions of the heart (e.g., the left ventricle). To capture the gross structure of the heart surface, the surgeon traced the beating heart with a probe equipped with an electromagnetic tracking sensor at the distal tip. The probe was inserted into the intrapericardial space through the subxiphoid incision. The position data recorded from the tracking probe were then used by the custom HeartLander graphic software to create a wire frame computer model of the heart surface. This tracing method did not account for the displacement or deformation of the heart surface caused by heartbeat and respiration, but provided only an approximation of the geometric shape of the heart surface. Because the heart surface model was defined with respect to the tracking system reference frame, it was automatically registered properly to the robot tracking sensor and the targets.

\section{Positioning Protocol}

The positioning study tested the ability of HeartLander to quickly and accurately acquire multiple targets located in a specified local pattern. A circular pattern, with a $15-\mathrm{mm}$ diameter, of seven evenly spaced targets and one central target was chosen for its clinical significance in defining a boundary (Fig. 5). The number of targets and target spacing were set by the surgeon based on the size and shape of the posterior left ventricle as determined from the heart surface model. The posterior left ventricle was selected for the target pattern due to its clinical significance in heart failure, and the difficulty with which it is accessed using conventional endoscopic approaches. The surgeon set the acceptable error range for target acquisition to $1.0 \mathrm{~mm}$, which was measured by the control system as the distance between the 3-D planned target location and the location of the robot tracking sensor. This level of accuracy was considered to be sufficient for direct epicardial injection therapy for heart 
failure. After each successful target acquisition, the front body was locked onto the epicardium with active suction and the surgeon performed an injection of dye into the myocardium with the remote injection system. The surgeon proceeded in this manner until all targets in the pattern were acquired and marked with dye. In addition to demonstrating injection capability, the dye marks served as visible landmarks showing the location of the robot following target acquisition. These marks were used to evaluate the accuracy of the positioning system on the excised porcine heart following the trial.

\section{Postmortem Study}

The animal was euthanized at the end of the trial, in accordance with the acute protocol approved for the study. The heart was first examined in situ to assess damage to non-cardiac structures, and was then removed. Gross visual inspection was performed from the epicardial vantage to surrounding structures in the mediastinum. The dye mark locations were photographed, and the regions of heart tissue at the injection sites were assessed for histological injury.

\section{RESULTS}

The animal tolerated the testing well until planned euthanasia. No adverse hemodynamic or electrophysiological events (e.g., hypotension, fatal arrhythmia, bleeding) were noted during the trial. The surrounding structures were intact upon postmortem examinations. There were no gross or histological injuries to the heart due to the robot motion, suction prehension, or injections.

The surgeon completed the heart surface tracing in approximately 5 minutes, while viewing the resulting point cloud generation on the graphic display of the user interface to ensure sufficient coverage. The resulting wire frame heart model created by the graphic display software contained sufficient geometric information of the heart surface for the surgeon to identify the general anatomical regions of the heart.

The surgeon was able to use the HeartLander system to administer dye injections to all target locations within the circular pattern on the posterior left ventricle. The target acquisitions were performed solely with motions of the front body, and did not require steps to reposition the rear body. The average acquisition time for each individual target was $23 \pm 15$ seconds, excluding the time required for dye injection. Following each target acquisition, an injection of $0.1 \mathrm{ml}$ of oil-based dye was injected into the myocardium using the remote injection system through the working channel of the HeartLander crawler. With the needle penetration depth set to $3 \mathrm{~mm}$, the average dye penetration depth was $3.0 \pm 0.5 \mathrm{~mm}$. The duration of the entire target acquisition and injection testing protocol was 25 minutes.

In evaluating the accuracy of the HeartLander system in the positioning task, we had two independent measures of the robot locations following target acquisition: the tracker-

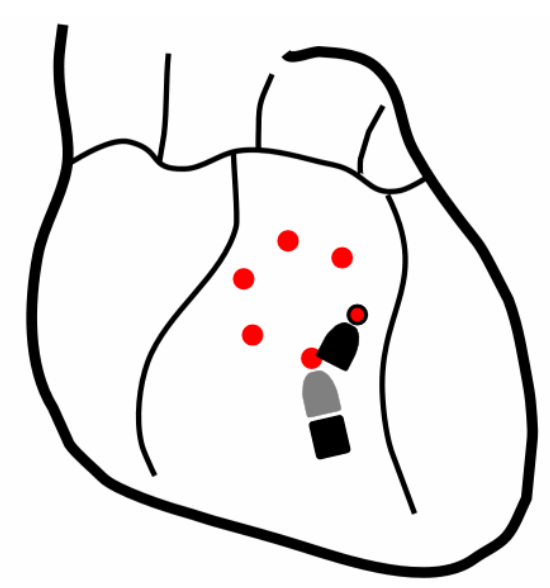

Fig. 5. Illustration of the positioning task. The robot makes a series of precise motions of the front body to each of the targets in the specified pattern, shown by the red circles. The initial location of the front body is shown in grey. The rear body remains stationary throughout the task.

based sensor positions measured by the control system, and the dye marks visible on the surface of the excised heart. In comparing these two measures to determine positioning accuracy, we considered both the absolute and relative components. We defined the "absolute" accuracy as the degree to which the target pattern was positioned and oriented in a similar manner between the tracker-based positions on the heart surface model, and the dye marks on the excised porcine heart. We defined the "relative" accuracy as the degree to which the shape of the target pattern was similar between the tracker-based measurements and dye marks on the porcine heart. Separating the accuracy into these independent components allowed us to make a more detailed assessment of the HeartLander positioning system.

\section{A. Absolute Accuracy}

The location and orientation of the tracker measurements and dye marks on the posterior heart surface were qualitatively compared in the assessment of absolute accuracy. The pattern of tracker measurements following the target acquisitions, along with the heart surface model, was oriented to display the posterior view of the heart (Fig. 6a). Similarly, the dye mark pattern and excised heart were photographed with posterior surface of the heart normal to the camera (Fig. 6b). The tracker image and dye photograph were scaled based on known distances in each image, and were oriented so the contours of the hearts aligned. Fig. $6 \mathrm{c}$ shows the tracker measurement image overlaid on the photograph of the excised heart. This figure demonstrates good qualitative agreement between the geometric shapes of the heart surface model and the excised heart. The figure also shows good qualitative agreement between the locations of the tracker measurements of the robot following the target acquisitions and the dye marks on the excised porcine heart. 


\section{B. Relative Accuracy}

The relative errors between the locations of the individual tracker measurements and dye marks were calculated in the assessment of the relative positioning accuracy. Four separate measurements of the target patterns were used in the calculation of the relative accuracy:

(1) the planned target locations defined with respect to the electromagnetic tracking system (method A),

(2) the tracker measurements of the robot immediately following target acquisition (method B),

(3) the tracker measurements of the robot during dye injection (method C), and

(4) the dye marks visible in photographs of the excised porcine heart (method D).

The difference in the robot state between methods B and C was that the front body did not grip the epicardium during the positioning motions (B), but did grip the epicardium during injection $(\mathrm{C})$. The planned target locations (A) and tracker measurements $(\mathrm{B}, \mathrm{C})$ were generated from a $2 \mathrm{D}$ projection of the tracker data with a view angle normal to the least-squares best-fit plane through the 3-D tracker data at the time of the dye injections (Fig. 7a). The dye mark locations (D) were generated using a photograph of the excised porcine heart with the camera oriented normal to the surface of the heart on which the dye pattern was visible (Fig. 7b). The location of the robot at each injection site was estimated as the center of the circular dye region on the tissue, which was validated in bench testing.

All tracker-based patterns and the dye mark photograph were scaled according to known distances in both images. The locations of the tracker-based patterns were properly registered to one another because they were all measured with respect to the tracking system reference frame. The dye mark pattern from the photograph was aligned with the tracker-based patterns by calculating the translation and rotation that minimized the sum of root-squared distances between the individual targets (Fig. 7c). This alignment of the tracker-based and dye mark patterns was reasonable as we were only considering the relative accuracy of the system in this analysis; i.e., the shape of the target patterns.

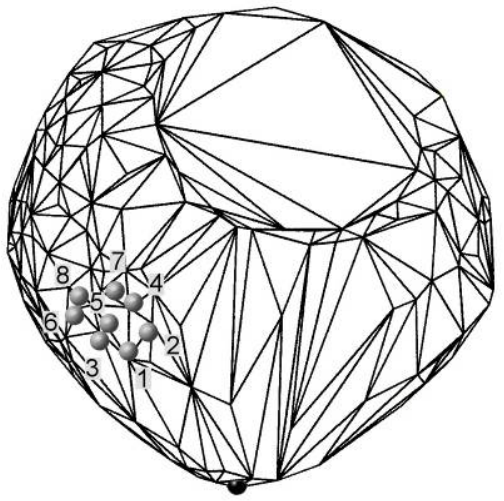

(a)

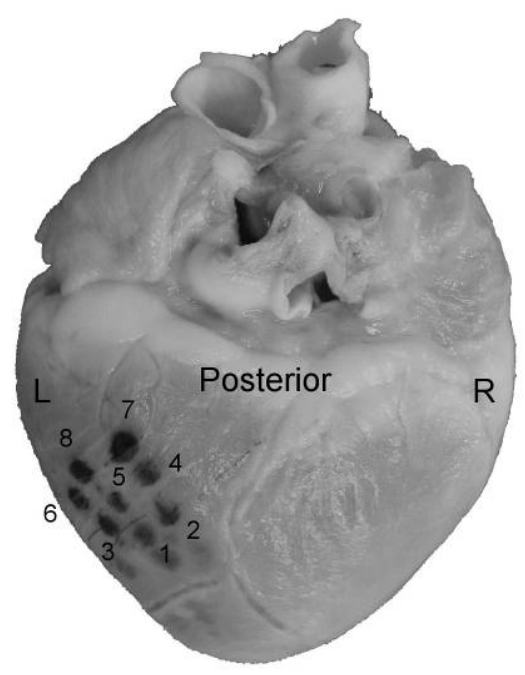

(b)

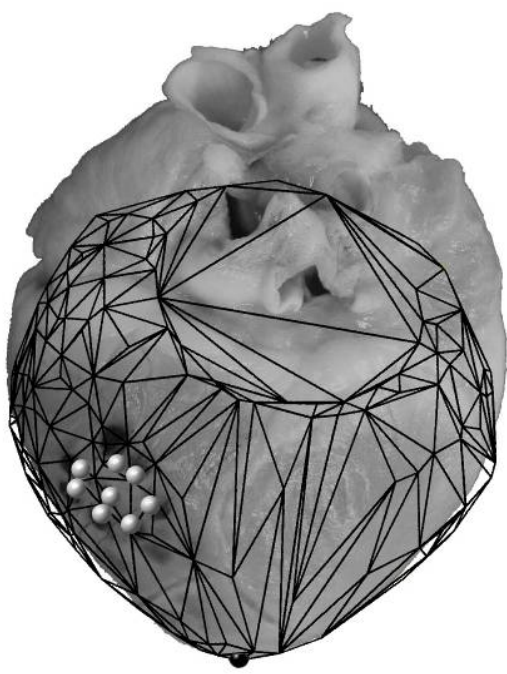

(c)

Fig. 6. (a) The heart surface model and tracker measurements of the robot sensor at the dye injections. (b) Photograph of the excised porcine heart and dye mark pattern. (c) The heart surface model and injection pattern overlaid on the injection photograph.

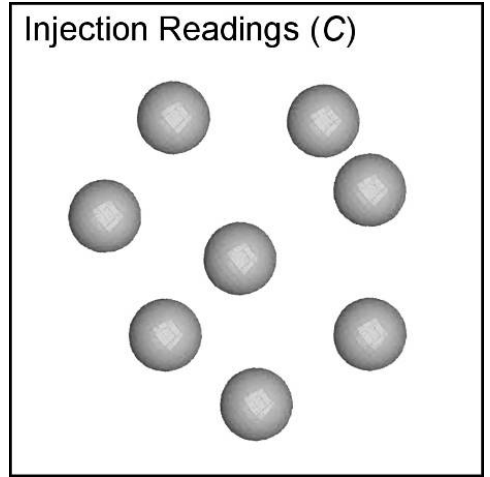

(a)

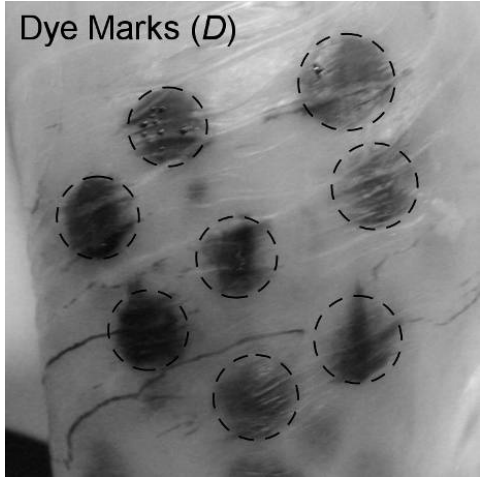

(b)

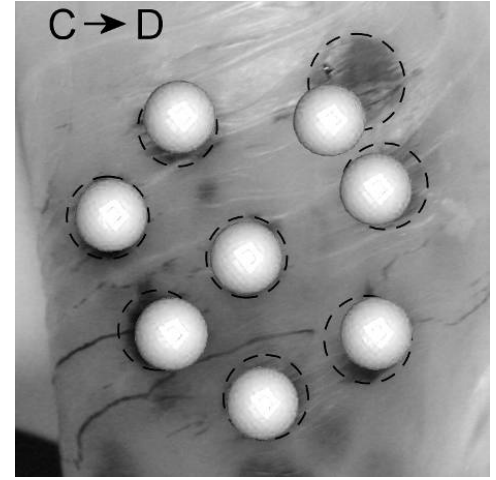

(c)

Fig. 7. (a) The tracker measurements of the robot sensor at the dye injections. (b) Photograph of the dye mark pattern (with each mark outlined) on the excised porcine heart. (c) The tracker measurements of the robot locations during the dye injections, overlaid on the dye mark pattern. 


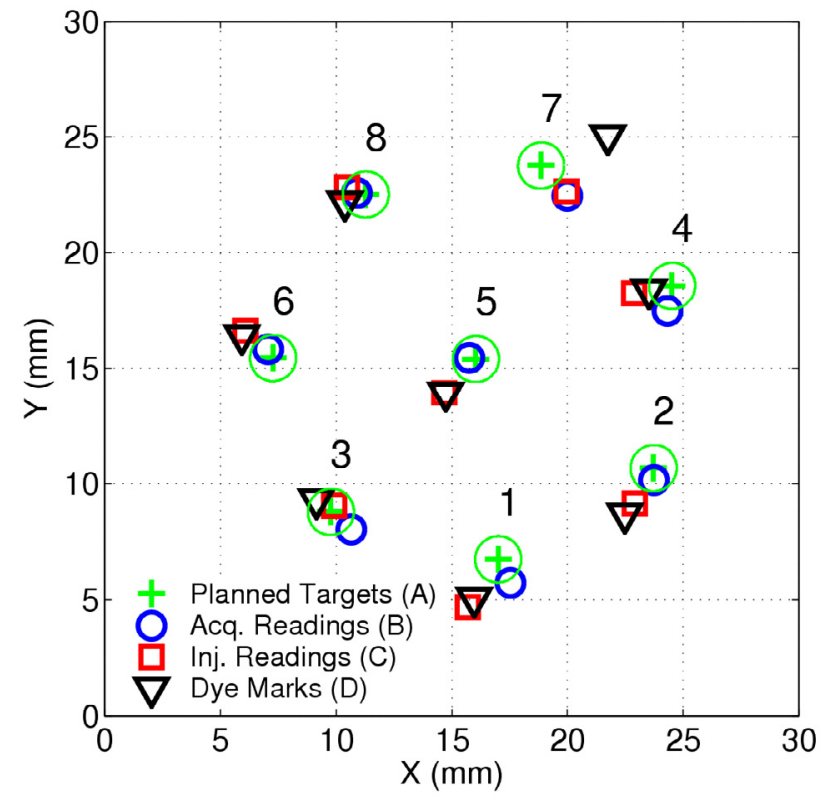

Fig. 8. A 2-D plot of the locations of all tracker-based patterns (planned targets, acquisition readings, and injection readings) and the dye marks.

TABLE I. THE VALUES OF EACH OF THE THREE SOURCES OF RELATIVE ERROR, AND THE TOTAL RELATIVE ERROR FOR THE POSITIONING TASK

\begin{tabular}{ccccccc}
\hline \hline $\begin{array}{c}\text { Target } \\
\text { No. }\end{array}$ & $\begin{array}{c}\text { Target } \\
\text { Distance } \\
(\mathrm{mm})\end{array}$ & $\begin{array}{c}\mathrm{A} \rightarrow \mathrm{B} \\
\text { Error } \\
(\mathrm{mm})\end{array}$ & $\begin{array}{c}\mathrm{B} \rightarrow \mathrm{C} \\
\text { Error } \\
(\mathrm{mm})\end{array}$ & $\begin{array}{c}\mathrm{C} \rightarrow \mathrm{D} \\
\text { Error } \\
(\mathrm{mm})\end{array}$ & $\begin{array}{c}\mathrm{A} \rightarrow \mathrm{D} \\
\text { Error } \\
(\mathrm{mm})\end{array}$ & $\begin{array}{c}\text { Duration } \\
(\mathrm{s})\end{array}$ \\
\hline 1 & 5.0 & 1.0 & 1.9 & 0.4 & 1.7 & 13 \\
2 & 9.8 & 0.4 & 0.8 & 0.6 & 2.3 & 28 \\
3 & 9.8 & 1.0 & 1.0 & 0.8 & 0.9 & 23 \\
4 & 15.9 & 0.9 & 1.6 & 0.7 & 0.3 & 9 \\
5 & 12.5 & 0.3 & 1.5 & 0.1 & 1.6 & 21 \\
6 & 15.9 & 0.4 & 1.1 & 0.3 & 1.9 & 20 \\
7 & 19.5 & 1.5 & 1.0 & 3.0 & 3.6 & 56 \\
8 & 5.0 & 0.3 & 0.3 & 0.7 & 1.1 & 11 \\
\hline $\begin{array}{c}\text { Mean } \\
\pm \text { Std }\end{array}$ & & $0.7 \pm 0.4$ & $1.1 \pm 0.5$ & $0.8 \pm 0.9$ & $1.7 \pm 1.0$ & $23 \pm 15$ \\
\hline
\end{tabular}

The total relative error between each planned target location and dye mark $(\mathrm{A} \rightarrow \mathrm{D})$ was the cumulative error between the planned target and the robot tracker at the target acquisition $(\mathrm{A} \rightarrow \mathrm{B})$, the robot tracker at the target acquisition and at the dye injection $(\mathrm{B} \rightarrow \mathrm{C})$, and the robot tracker at the dye injection and the physical dye mark on the heart tissue $(C \rightarrow D)$. All errors were calculated as the $2 \mathrm{D}$ resultant distances between the individual targets in the aligned images, and were reported individually and averaged over all targets. The 2D locations of the targets calculated using all four methods can be found in Fig. 8, while the individual and averaged error values are in Table I. The average total relative error from the planned targets to the dye marks on the heart tissue was $1.7 \pm 1.0 \mathrm{~mm}$.

\section{DISCUSSION}

In the present study, HeartLander demonstrated semiautonomous acquisition of a specified target pattern, followed by dye injections, on the posterior surface of a beating porcine heart through a subxiphoid approach with no adverse physiological events. It is noteworthy that surgeons have some difficulty accessing this surface even under full sternotomy. The absolute accuracy of the tracker measurements of the robot location and the photograph of the dye marks on the excised porcine heart was qualitatively good. This validates that the heart surface model serves as a geometric representation of the heart surface that agrees with the surface of the true porcine heart. It also validates the use of the surface model and electromagnetic tracking system to identify the general anatomical location of the robot on the true heart.

The relative accuracy between the planned target locations and the tracker measurements of the robot following target acquisition $(\mathrm{A} \rightarrow \mathrm{B})$ had an average value of $0.7 \mathrm{~mm}$, which was below the range set by the surgeon in the control system $(1.0 \mathrm{~mm})$. The total relative accuracy between the planned target locations and the dye marks $(A \rightarrow D)$, however, increased to an average of $1.7 \mathrm{~mm}$. Although this value exceeds the error limit set within the control system, these results demonstrate the most accurate positioning on the posterior beating heart surface through a minimally invasive approach known to these authors. The additional relative error from $\mathrm{B} \rightarrow \mathrm{C}$ was due to the gripping of the epicardium following target acquisition, which is measured by the tracking system and can therefore be reduced with modifications to the control system. The additional error from $\mathrm{C} \rightarrow \mathrm{D}$ may have resulted from the reported static inaccuracy of the electromagnetic tracking system $(1.4 \mathrm{~mm})$, or from uneven distribution of the dye into the myocardium. This issue must be explored in future work. It may be that the large error associated with target 7 was due to this uneven distribution of the dye into the myocardium, causing us to miscalculate the location of the front body during dye injection.

By adhering directly to the epicardium, and thus passively synchronizing with the heart, the 3-D locations of the tip of the injection needle and the epicardial surface remained aligned. Stabilization of the distance between the needle and the heart surface resulted in reproducible needle penetration depths, while stability tangential to the heart surface ensured that the needle did not tear the tissue. Injection methods not synchronizing with the heart motion (e.g., manual or endoscopic tool injection) suffer with regard to both of these issues. In this manner, HeartLander does not require cardiac stabilization for injection, which significantly reduces the risks of hemodynamic impairment and fatal arrhythmia that can result from the use of commercial mechanical stabilizers to immobilize the surface of the heart. Filtering the physiological motion from the tracking data does not, however, address the local deformation between points on 
the heart surface that occur during the cardiac cycle. Despite this fact, we were able to demonstrate high relative positioning accuracy $(1.7 \mathrm{~mm})$ because these deformations decrease as the distance between the robot and the current target decreases. In the future, this physiological deformation error can be further reduced by gating the tracking data - using real-time ECG and air flow measurements - so that only data collected during specified phases of the cardiac and respiratory cycles are used in the control system.

The most crucial limitation of our current system is the lack of anatomical information in the graphic display of the user interface. This deficit presents a serious safety concern when navigating on and interacting with the epicardial surface, and reduces our ability to define the absolute positioning accuracy of the HeartLander system. Accordingly, one of our future research aims is to register preoperative anatomical imaging (e.g. CT or MRI) with realtime tracking techniques. The surface model presented in this study was only used by the surgeon to estimate the general anatomical location of the robot based on the known geometry of the heart surface. Similarly, the positioning targets were defined relative to one another, rather than to specific anatomical landmarks on the epicardium. In the future, it will be necessary to quantitatively evaluate the absolute accuracy of the positioning system. This evaluation will become possible with proper registration of preoperative imaging and real-time tracking.

There are myriad opportunities for therapeutic applications within the intrapericardial space; including, but not limited to, cell transplant therapy for heart failure, left atrial appendage ligation, epicardial ablation, device-based mitral valve repair, and epicardial pacemaker lead placement for cardiac resynchronization therapy [2, 14, 15]. Recently, several minimally invasive approaches, such as traditional and robotic-assisted thoracoscopy, have been reported for these therapies [8, 16, 17]. These approaches, however, require multiple port placements under general anesthesia with double lumen ventilation. In addition, commercially available rigid endoscopic instruments intrinsically limit the operative field; specifically, it is difficult to access the posterior of the heart, and changing operative sites may require additional incisions and reinsertion of instrumentation. Alternatively, the insertion of HeartLander employs a subxiphoid approach. This approach is a useful method to access the intrapericardial space because there are no significant anatomic barriers, and it requires only a single port. Deploying a robot with navigation and positioning capabilities through this approach has the potential to obviate general endotracheal anesthesia, lung deflation, and full sternotomy without sacrificing treatment efficacy. In this manner, HeartLander has the potential to enable cardiac procedures on an outpatient basis.

\section{REFERENCES}

[1] S. A. Hunt et al., "ACC/AHA 2005 Guideline Update for the Diagnosis and Management of Chronic Heart Failure in the Adult: a report of the American College of Cardiology/American Heart Association Task Force on Practice Guidelines," Circulation, vol. 112, pp. e154-235, Sep 202005.

[2] K. L. Christman and R. J. Lee, "Biomaterials for the treatment of myocardial infarction," J Am Coll Cardiol, vol. 48, pp. 907-13, Sep 5 2006.

[3] N. F. Huang, J. Yu, R. Sievers, S. Li, and R. J. Lee, "Injectable biopolymers enhance angiogenesis after myocardial infarction," Tissue Eng, vol. 11, pp. 1860-6, Nov-Dec 2005.

[4] B.-U. Kohler, C. Hennig, and R. Orglmeister, " The principles of software QRS detection," Engineering in Medicine and Biology Magazine, IEEE, vol. 21, pp. 42-57, 2002.

[5] D. Orlic, J. Kajstura, S. Chimenti, I. Jakoniuk, S. M. Anderson, B. Li, J. Pickel, R. McKay, B. Nadal-Ginard, D. M. Bodine, A. Leri, and P. Anversa, "Bone marrow cells regenerate infarcted myocardium," Nature, vol. 410, pp. 701-5, Apr 52001.

[6] A. Z. Segal, W. B. Abernethy, I. F. Palacios, R. BeLue, and G. Rordorf, "Stroke as a complication of cardiac catheterization: risk factors and clinical features," Neurology, vol. 56, pp. 975-7, Apr 10 2001.

[7] T. Freyman, G. Polin, H. Osman, J. Crary, M. Lu, L. Cheng, M Palasis, and R. L. Wilensky, "A quantitative, randomized study evaluating three methods of mesenchymal stem cell delivery following myocardial infarction," Eur Heart J, vol. 27, pp. 1114-22, May 2006.

[8] H. C. Ott, J. Brechtken, C. Swingen, T. M. Feldberg, T. S. Matthiesen, S. A. Barnes, W. Nelson, and D. A. Taylor, "Robotic minimally invasive cell transplantation for heart failure," J Thorac Cardiovasc Surg, vol. 132, pp. 170-3, Jul 2006.

[9] N. A. Patronik, M. A. Zenati, and C. N. Riviere, "Preliminary evaluation of a mobile robotic device for navigation and intervention on the beating heart," Comput Aided Surg, vol. 10, pp. 225-32, Jul 2005

[10] C. N. Riviere, N. A. Patronik, and M. A. Zenati, "Prototype epicardial crawling device for intrapericardial intervention on the beating heart," Heart Surg Forum, vol. 7, pp. E639-43, 2004.

[11] N. A. Patronik, T. Ota, M. A. Zenati, and C. N. Riviere, "A miniature mobile robot for precise and stable access to the beating heart," IEEE Transactions on Robotics, submitted for publication.

[12] M. Schneider and C. Stevens, "Development and testing of a new magnetic-tracking device for image guidance," Proceedings of SPIE Medical Imaging 2007: Visualization and Image-Guided Procedures, vol. 6505, p. 65090I, 2007.

[13] C. Borst, E. W. Jansen, C. A. Tulleken, P. F. Grundeman, H. J Mansvelt Beck, J. W. van Dongen, K. C. Hodde, and J. J. Bredee, "Coronary artery bypass grafting without cardiopulmonary bypass and without interruption of native coronary flow using a novel anastomosis site restraining device ("Octopus")," J Am Coll Cardiol, vol. 27, pp. 1356-64, May 1996.

[14] R. Cappato, H. Calkins, S. A. Chen, W. Davies, Y. Iesaka, J. Kalman, Y. H. Kim, G. Klein, D. Packer, and A. Skanes, "Worldwide survey on the methods, efficacy, and safety of catheter ablation for human atrial fibrillation," Circulation, vol. 111, pp. 1100-5, Mar 82005.

[15] M. Rivero-Ayerza, D. A. Theuns, H. M. Garcia-Garcia, E. Boersma M. Simoons, and L. J. Jordaens, "Effects of cardiac resynchronization therapy on overall mortality and mode of death: a meta-analysis of randomized controlled trials," Eur Heart J, vol. 27, pp. 2682-8, Nov 2006.

[16] J. C. Pruitt, R. R. Lazzara, G. H. Dworkin, V. Badhwar, C. Kuma, and G. Ebra, "Totally endoscopic ablation of lone atrial fibrillation: initial clinical experience," Ann Thorac Surg, vol. 81, pp. 1325-30; discussion 1330-1, Apr 2006.

[17] M. A. Zenati, "Robotic heart surgery," Cardiol Rev, vol. 9, pp. 287-94, Sep-Oct 2001 